Chronic Obstructive Pulmonary Diseases: Journal of the COPD Foundation

\author{
Brief Report
}

\title{
The COPD Foundation Coronavirus Disease 2019 International Medical Experts Survey: Results
}

\author{
Ruth Tal-Singer, $\mathrm{PhD}^{1}$ Barbara P. Yawn, MD ${ }^{1,2}$ Robert Wise, $\mathrm{MD}^{3}$ Byron M. Thomashow, MD ${ }^{1,4}$ \\ Danielle M. Boyce, $\mathrm{MPH}^{1}$
}

\begin{abstract}
Abbreviations: severe acute respiratory syndrome coronavirus 2, SARS-CoV-2; coronavirus disease 2019, COVID-19; Medical and Scientific Advisory Committee, MASAC; chronic obstructive pulmonary disease, COPD; World Health Organization, WHO; Centers for Disease Control and Prevention, CDC; acute respiratory distress syndrome, ARDS; Middle East respiratory syndrome, MERS; severe acute respiratory syndrome, SARS

Funding Support: not applicable

Date of Acceptance: May 15, 2020

Citation: Tal-Singer R, Yawn B, Wise B, Thomashow B, Boyce DM. The COPD Foundation Coronavirus Disease 2019 International Medical Experts Survey: results. Chronic Obstr Pulm Dis. 2020;7(3):139-146. doi: https://doi.org/10.15326/jcopdf.7.3.2020.0164
\end{abstract}

1 COPD Foundation, Research Department, Washington, DC

2 University of Minnesota, Family and Community Health, Minneapolis

3 Pulmonary and Critical Care, Department of Medicine, Johns Hopkins University School of Medicine, Baltimore, Maryland

4 Division of Pulmonary, Allergy, and Critical Care Medicine, Columbia University, New York, New York

\section{Address correspondence to:}

Ruth Tal-Singer, PhD

Email: rtalsinger@copdfoundation.org

Phone: 610-570-4876

\section{Keywords:}

coronavirus disease 2019; COVID-19; medical experts; steroids; discharge

\section{Introduction}

The COPD Foundation has been monitoring the global pandemic of a disease caused by the recently identified coronavirus family member severe acute respiratory syndromve coronavirus 2 (SARS-CoV-2) named coronavirus disease 2019 (COVID-19). The Foundation's Research team has been working with the Foundation's Medical and Scientific Advisory Committee (MASAC) ${ }^{1}$ to ensure that accurate information is shared with the chronic obstructive pulmonary disease (COPD) community. ${ }^{2}$
The spread of COVID-19 is a fast-moving, constantly evolving situation with limited peerreviewed evidence. Using Survey Monkey, we conducted 2 anonymous surveys administered to pulmonary medicine experts. The pilot 5-question survey was administered over the course of just 2 days (March 15-16, 2020) to 19 members of the COPD Foundation MASAC with the aim to explore expert opinion on the use of corticosteroids when treating COPD patients with COVID-19. The second survey was administered between April 17th and April 26th, 2020 and included 18 MASAC respondents as well as 10 experts from the COPD Foundation's extended global expert collaborator network ("Non-MASAC"). In addition to repeating the 5 questions from Survey 1 , the second survey explored expert opinion on other issues related to the treatment of hospitalized individuals with COVID-19.

\section{Systemic Steroid Treatment in COPD Outpatients With or Without Coronavirus Disease 2019 (2 questions)}

Questions: (1) "Should we recommend prescribing prednisone for COPD outpatients, who have an exacerbation bad enough that it would normally be treated with prednisone, where coronavirus status 
is unclear?" (2) "Should we recommend prescribing prednisone for COPD outpatients, who have an exacerbation bad enough that it would normally be treated with prednisone, with proven COVID-19?"

The results of Yes/No responses to both surveys are presented in Table 1 . In response to questions on prescribing systemic steroids to patients being treated as outpatients for a COPD exacerbation regardless of COVID-19 status, analysis of text responses indicated that most respondents in both surveys preferred to follow existing treatment guidelines. At the time of the pilot survey, which preceded COPD-specific guidance, ${ }^{3}$ respondents reported lack of evidence on steroid therapy being harmful but recognized that at the time the World Health Organization (WHO) and the U.S. Centers for Disease Control and Prevention (CDC) recommended tempering use of systemic steroids. In the second survey, the majority of respondents answered "Yes" to these questions and there was no difference between MASAC and Non-MASAC respondents. Respondents cited lack of evidence against using steroids and indicated a preference to follow the current guidance for management of a COPD exacerbation. ${ }^{4}$ One respondent suggested the use of lower/minimally effective doses should be considered.
Those who disagreed expressed concerns that steroids promote infection; one respondent mentioned specific concern about using steroids in the early phase of COVID-19 and might consider steroids later in the "hyper inflammatory" phase.

\section{Systemic Steroid Treatment in Hospitalized COPD Patients With Coronavirus Disease 2019}

Questions: (3) "Should we recommend prescribing systemic steroids for hospitalized COPD patients proven to have COVID-19 with an exacerbation without infiltrates?" and (4) "Should we recommend prescribing systemic steroids for a hospitalized individual with COPD with COVID-19 with infiltrates?"

The majority of respondents stated that systemic steroids would be the recommended treatment if the patient was presenting with a COPD exacerbation (wheezy, bronchospastic) rather than COVID-19 pneumonitis (hypoxia, acute lung injury, acute respiratory distress syndrome $[A R D S])$. They noted that there is currently limited evidence to the contrary, and steroids are used safely and effectively in other virus-induced COPD exacerbations. A few individuals

\section{Table 1. Responses to Repeated Questions in Survey 1 and Survey 2}

Question

\begin{tabular}{|c|c|c|c|}
\hline $\begin{array}{c}\text { Survey } 1 \\
\% \text { Yes }\end{array}$ & $\begin{array}{c}\text { Survey } 2 \\
\% \text { Yes }\end{array}$ & $\begin{array}{c}\text { Survey } 1 \\
\% \text { No }\end{array}$ & $\begin{array}{c}\text { Survey } 2 \\
\% \text { No }\end{array}$ \\
$(\mathrm{N})$ & $(\mathrm{N})$ & $(\mathrm{N})$ & $(\mathrm{N})$
\end{tabular}

$89.5 \%$

(17) $74 \%$

(14) $74 \%$

(14)

$53 \%$

(10)

$79 \%$

(15)

steroids in COPD patients who are not frequent exacerbators especially those with low eosinophil counts, should we make that recommendation even stronger in face of present pandemic where early data suggests that those infected are more likely to be eosinopenic? (understanding lack of COPD specific data).

93\%

(26)

$10.5 \%$

(2)

$89 \%$

(25)

$26 \%$

(5)

$86 \%$

(24)

26\%

(5)

$14 \%$

(4)

without infiltrates?

should we recommend systemic steroids for an exacerbation with infiltrates?

(5) Recognizing that most of us have discouraged use of inhaled

,

西

$57 \%$

(16)

$57 \%$

(16)

MASAC

only

$61 \%(11)$
$7 \%$

(2)
$11 \%$

(3)

COVID-19=coronavirus disease 2019; $\mathrm{COPD}=$ chronic obstructive pulmonary disease; MASAC=Medical and Scientific Advisory Committee 
suggested treating on a case-by-case basis based on clinical judgment: withhold steroids if the patient presents with low blood eosinophil count or a bacterial infection. If prescribed, one has to accept the risk for prolonged viral shedding and use appropriate containment methods. Some respondents noted the lack of data to support a recommendation for prescribing steroids in a hospitalized patient ${ }^{5}$ and that there is mixed information for prescribing steroids for ARDS based on prior Middle East respiratory syndrome (MERS) and severe acute respiratory syndrome (SARS) experience. ${ }^{6}$ A comment from one respondent who answered "No" stated that there is no evidence that systemic steroids are helpful for an exacerbation without infiltrates in a patient with COPD and proven COVID-19.

There appeared to be less consensus in the responses to Question 4, which asked about prescribing systemic steroids for hospitalized COPD patients with COVID-19 and pulmonary infiltrates (Figure 1). However, upon review of open-ended comments, it became clear that several respondents in each category required more details about the case (e.g., whether or not symptoms are present, the primary driver of hospitalization, and disease pathology) to definitively answer "yes" or "no".

It is possible that respondents in both groups might have answered "maybe" if that choice was offered. Both "yes" and "no" respondents emphasized the need to proceed with caution. An additional "yes" comment highlighted the need for shared decision making when assessing risks and benefits and the need to treat COPD exacerbations with standard of care ("Treat like you would any acute exacerbation of COPD"). The overwhelming remainder of the "No" comments emphasized the lack of evidence or conclusive data to make this recommendation.

\section{Inhaled Steroid Treatment in COPD Patients with Low Blood Eosinophil Count During Coronavirus Disease 2019 Pandemic}

Question: "Recognizing that most of us have discouraged use of inhaled steroids in COPD patients who are not frequent exacerbators, especially those with low eosinophil counts, should we make that recommendation even stronger in the face of the present pandemic where early data suggests that those infected are more likely to be eosinopenic? (understanding lack of COPD-specific data)."

In both surveys, the quantitative results led us to believe that the group is polarized on this topic. However, upon review of open-ended comments, it is evident that both sides agree with the existing recommendation to discourage the use of inhaled steroids in COPD patients who are not frequent exacerbators, especially those with low blood eosinophil counts. Respondents do not seem to feel strongly about updating or strengthening this

\section{Figure 1. Responses to the Question, "In hospitalized patients with COPD and proven COVID-19, should we recommend systemic steroids for an exacerbation with infiltrates?"}

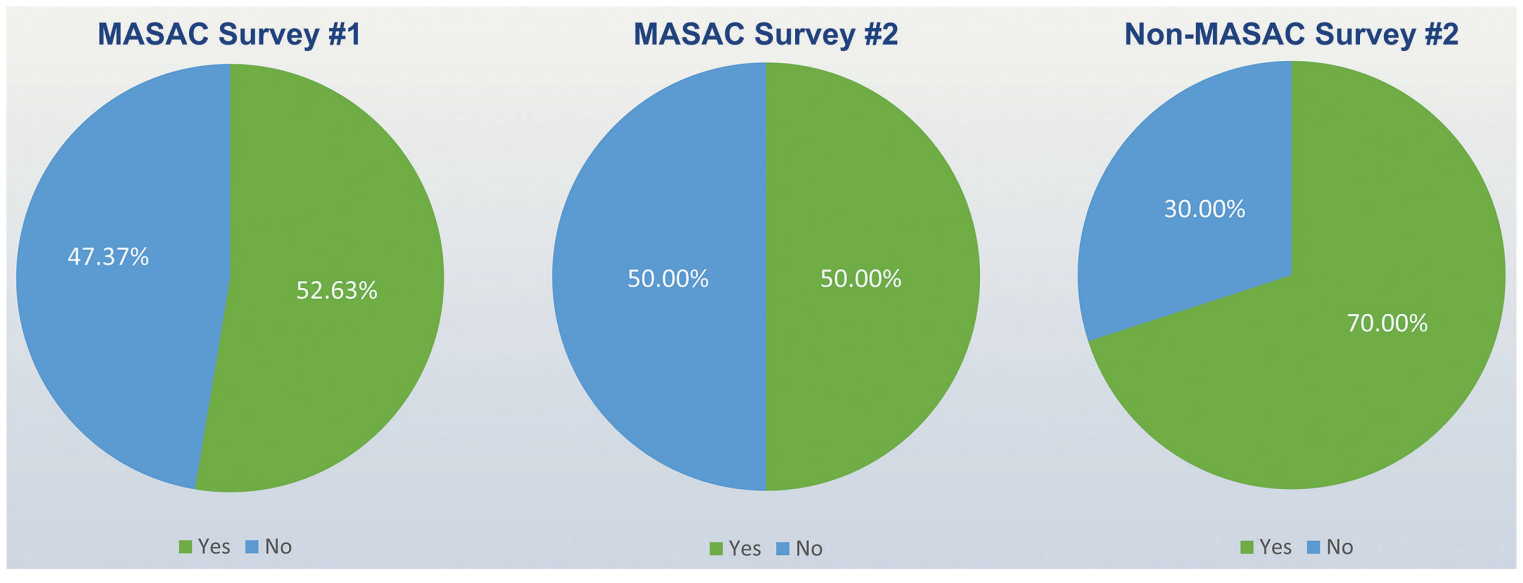

This figure highlights the consistency of MASAC responses in both surveys. Blue represents "No" and green represents "Yes" responses. 
recommendation, as even those who answered "yes" (to strengthen the recommendation) didn't seem to feel very strongly about it based on their comments:

- Yes: "I am generally a strong advocate of deimplementing ICS (inhaled corticosteroids) and this is just another reason to do so."

- No: "I think we should continue present recommendations, but don't think we have the data to yet make that recommendation even stronger."

\section{Discharge From the Hospital Following Coronavirus Disease 2019 Admission}

Question: "Assuming clinical stability and limited available hospital beds, which of the following would you consider the strongest criterion for planning a COVID-19 patient's hospital discharge?"

Fifty percent (14) of respondents relied on a resting room air oxygen saturation of at least $92 \%$ to support hospital discharge (Figure 2). The majority of respondents who provided comments based the

Figure 2. Responses to the Question, "Assuming clinical stability and limited available hospital beds, which of the following would you consider the strongest criterion for planning a COVID-19 patient's hospital discharge?"

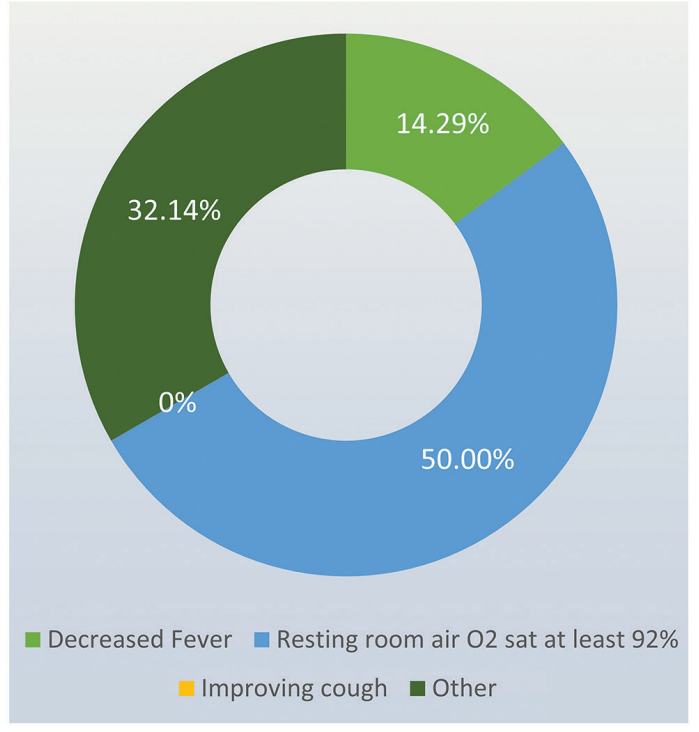

This figure demonstrates "resting room air oxygen saturation of at least $92 \%$ " as the strongest criterion for planning a COVID-19 patient's hospital discharge among $50 \%$ of the survey respondents. decision on a combination of circumstances that factor into the decision to plan hospital discharge, including a positive overall clinical status and the ability of the patient to maintain their safety at home with an adequate support system (assistance with daily functions, shopping, errands, etc.). No respondent selected the option "improved cough" as a discharge criterion.

In response to a question about their preferred patient follow-up approach, $80 \%$ of respondents selected the option of "telemedicine plus technology supporting assessment of oximetry, vitals, and symptoms" (Figure 3). There was less interest in a mobile stethoscope or assessing lung function although the text comments indicated no experience or familiarity with these options.

\section{Moving to Tracheostomy in Intubated Coronavirus Disease 2019 Patients}

Question: "At what point are you moving to tracheostomy in intubated COVID-19 patients?"

Three-quarters of respondents $(75 \%, n=21)$ indicated that they are moving to tracheostomy measures after a patient is on a ventilator for 2 or more weeks. None of the respondents indicated that they are moving to tracheostomy measures "less than 1 week on the ventilator." Many respondents indicated the preference to avoid tracheostomy due to the highrisk nature of this procedure. A comment from 1 of 5 respondents who responded "1-2 weeks on ventilator" indicated that "patients tend to get worse after 1 week on a ventilator."

\section{Anticoagulation Regimens in Coronavirus Disease 2019 Patients (Hospital)}

Question: "Recognizing the concern that a significant number of COVID-19 hospitalized patients are prothrombotic and/or have markedly elevated D-dimer levels, which of the following anticoagulation regimens would you support using in COVID-19 patients without documented VTE and no contraindication for anticoagulation?”

A total of $68 \%$ of Survey 2 respondents (19) selected the option of "standard thromboprophylaxis dosing with enoxaparin, if not limited by renal failure or HITT" and 25\% (7) selected "use D-dimer level to decide if need to treat with thromboprophylaxis or full 


\section{Figure 3. Responses to the Question, "How would you prefer to manage a COVID-19 patient following discharge from the hospital?"}

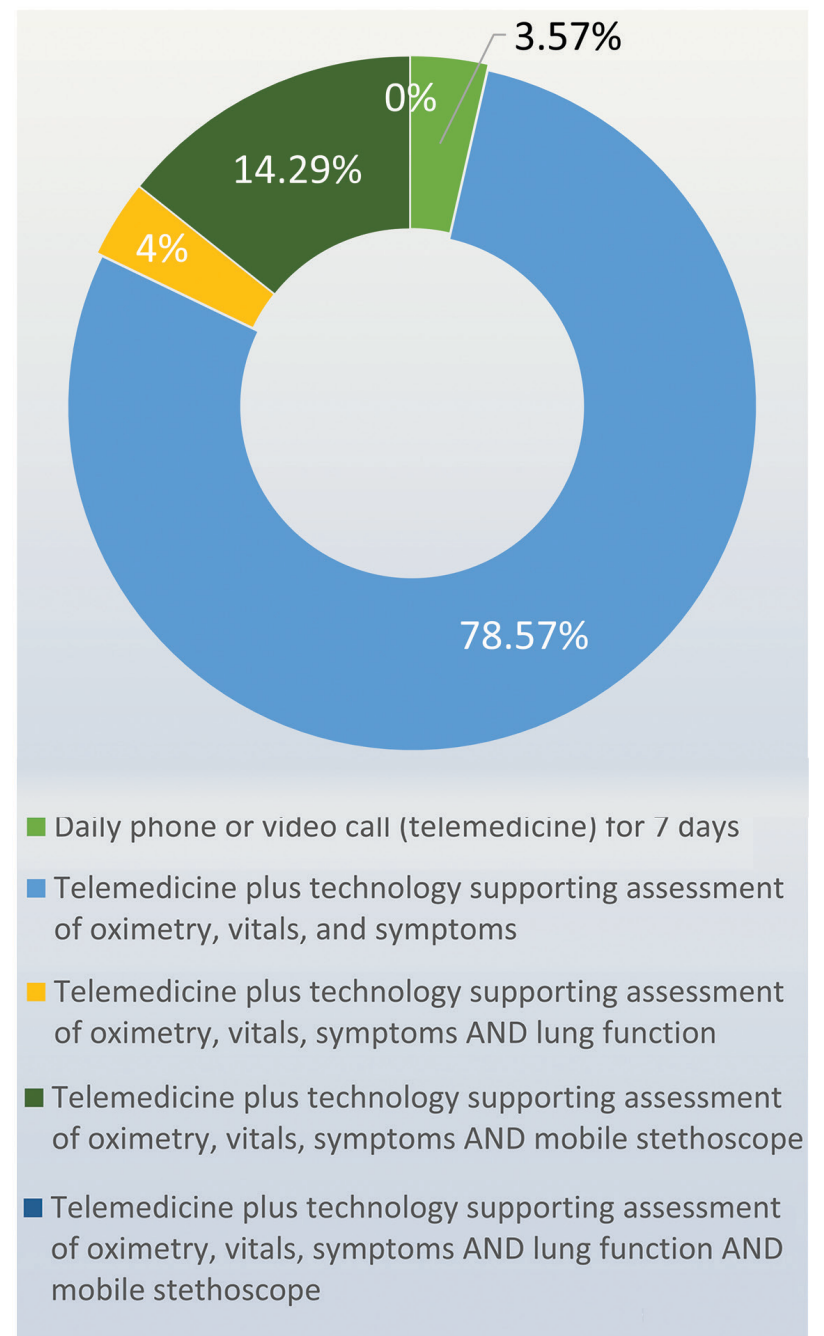

This figure demonstrates "telemedicine plus technology supporting assessment of oximetry, vitals, and symptoms" as the majority of survey respondents' preferred method of managing a COVID-19 patient following discharge from a hospital.

dose anticoagulation (e.g., if $\mathrm{D}$-dimer $>6$ times the upper limit of reference range, recommend treating with full dose anticoagulation with enoxaparin, unless contraindicated)." Several respondents stated in the text comments that the D-dimer protocol may be compelling, but most feel that the evidence is insufficient to choose anything other than standard approaches. Only one respondent selected the answer option stating "standard thromboprophylaxis dosing with unfractionated heparin." These responses are consistent with a recent consensus statement ${ }^{7}$ discussed on MedPage Today published on April 30, $2020 .^{8}$

\section{Anticoagulation Regimens in Coronavirus Disease 2019 Patients (Discharge)}

Question: "There have been some reports of increased risk of pulmonary embolism in COVID-19 patients post discharge. Which of the following would you suggest?"

There have been some reports of increased risk of pulmonary embolism in COVID-19 patients post discharge. ${ }^{9}$ Thirty-six percent of respondents (10) selected the option "discharge on thromboprophylaxis if no clear bleeding risk," one respondent selected "discharge on thromboprophylaxis if no clear bleeding risk depending on pre-discharge D-dimer levels" and 3 respondents would arrange routine leg ultrasounds pre-discharge if resources allowed, then decide about thromboprophylaxis. Thirteen respondents answered, "hope for the best." Qualitative analysis of 12 text responses revealed that this sentiment is largely due to lack of evidence for any specific protocol.

\section{Addressing Mucus Plugging in Ventilated Coronavirus Disease 2019 Patients}

Question: How do you address mucus plugging in intubated COVID-19 patients?

As shown in Table 2, most respondents selected the heated humidifier option. It is important to note that there are currently available infection control strategies that include closed systems that reduce the risk of aerosols associated with commonly used heat and moisture exchangers (e.g., Fisher and Paykel Evaqua $\left.{ }^{\mathrm{TM}}\right){ }^{10}$ One respondent suggested bronchoscopy as a last resort, and others commented on the importance of adequate hydration.

\section{Estimating Survival Rates and Cause of Death in Hospitalized Coronavirus Disease 2019 Patients}

Question: "Based on your experience, what is the survival rate of COPD patients hospitalized with COVID-19? What are the causes of death?" 


\section{Table 2. Responses to "How Do You Address Mucus Plugging in Intubated COVID-19 Patients? Select All That Apply"}

\begin{tabular}{|c|c|c|}
\hline Answer & \multicolumn{2}{|c|}{ Count } \\
\hline \multirow{2}{*}{ Treat with anti-mucolytics } & No & $19(68 \%)$ \\
\hline & Yes & $9(32 \%)$ \\
\hline \multirow{2}{*}{$\begin{array}{l}\text { Use a high frequency chest wall } \\
\text { oscillation device }\end{array}$} & No & $19(68 \%)$ \\
\hline & Yes & $9(32 \%)$ \\
\hline \multirow{2}{*}{$\begin{array}{l}\text { Adding heated humidifier } \\
\text { to ventilator }\end{array}$} & No & $13(46 \%)$ \\
\hline & Yes & $15(54 \%)$ \\
\hline \multirow{2}{*}{$\begin{array}{l}\text { Other (narrative comment for "other" } \\
\text { was to bronchoscopy as a last resort } \\
\text { option) }\end{array}$} & No & $20(71 \%)$ \\
\hline & Yes & $8(29 \%)$ \\
\hline
\end{tabular}

Based on close-ended responses, $65 \%$ of respondents marked $50 \%$ or greater survival. Some respondents indicated that they do not have enough experience with COVID-19 to provide a definitive answer. One respondent attributed the fact that they have not seen any COVID-19-related deaths due to adequate staffing and other resources at their hospital. Causes of death listed were mostly (Figure 4) multi-system organ failure (50\%), cardiac causes (1 respondent) and ARDS/respiratory failure (9 or 34.6\%). Three text responses added ARDS with sepsis, infectious complications, and sudden death; one respondent stated, "all of the above."

\section{Factors Informing a Decision to Send a Patient with Chronic Respiratory Disease for Coronavirus Disease 2019 Testing}

Question: "In a recent COPD360social COVID-19 survey, we noticed many COPD exacerbations since January 2020, but few patients were tested for COVID-19. What factors would contribute to your decision to send a patient with a chronic respiratory disease for COVID-19 testing?"

In a recent COPD360social COVID-19 community survey, ${ }^{11} 177$ patients with COPD reported exacerbations since January 2020, but only 6 patients were tested for COVID-19. This question explored factors that would contribute to a physician's decision to send a patient with a chronic respiratory disease for COVID-19 testing. There were 8 open-ended

\section{Figure 4. Responses to Question, "In those who died (COVID-19 patients), what was the cause of death?"}

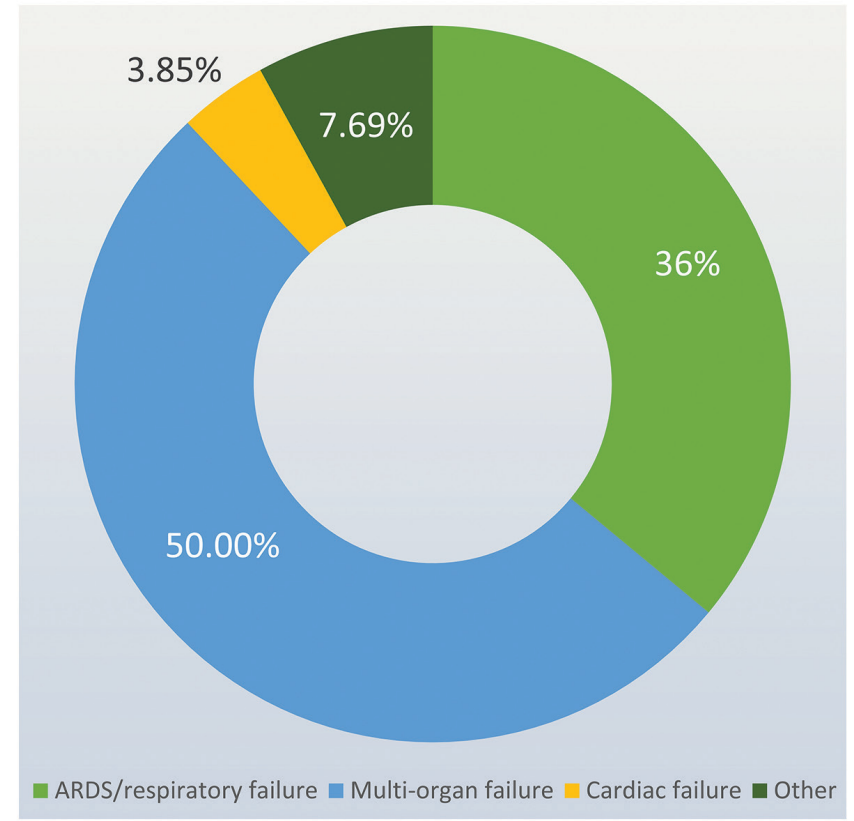

This figure demonstrates the cause of death (in COVID-19 patients) reported by survey respondents. The total ' $n$ ' of 26 includes those who selected a multiple choice answer as well as those who only provided a text response.

responses that elaborated on the "other" answer option. Of those, 6 expressed concerns about lack of availability of testing and lack of information and data due to insufficient validated testing approaches. One respondent expressed concern about sending an outpatient COPD patient to a COVID-19 testing site (presumably due to the risk of potential exposure). One respondent indicated that they would test if the patient indicated that this was an atypical exacerbation, i.e., had "GI symptoms and/or loss of taste or smell."

\section{Summary}

Overall, given the lack of evidence, most participants in these 2 surveys erred on the side of following existing recommendations ${ }^{3,4}$ for treating COPD patients during the COVID-19 pandemic. Many survey participants perceived an element of risk associated with managing patients with COPD when COVID-19 is proven or suspected. Unlike other viral infections, participants have limited clinical experience in treating people with COPD who have proven or suspected 
COVID-19, which is consistent with limited published data on COPD patients. ${ }^{12,13}$ Shared decision-making can help patients and health care professionals to navigate the risks of various treatment options. Several participants expressed concern that current testing for COVID-19 is inadequate. The responses on the telehealth question reflected a lack of familiarity or experience with novel eHealth tools. Specifically, given the limited experience of rapidly emerging technology to assess lung function with effort-free sensors and evaluate breath sounds or cough using voice analysis algorithms, it would be sensible to evaluate the impact and cost benefit of additional devices.

A key limitation of this study is the small sample size of a select group of pulmonary medicine specialists who collaborate with the COPD Foundation. If it is deemed useful, the COPD Foundation COVID-19 physician surveys may be expanded, enhanced and repeated during the period of this pandemic.

We encourage readers to provide us with feedback or submit questions of interest that could be administered in future surveys. Please direct all feedback and/ or questions to Ruth Tal-Singer, Chief Scientific Strategy Officer, COPD Foundation, rtalsinger@ copdfoundation.org.

\section{Declaration of Interest}

Dr. Tal-Singer is a former employee and current shareholder of GlaxoSmithKline and has received personal fees from Vocalis Health and Immunomet outside of the submitted work. Dr. Yawn reports personal fees and non-financial support from Boehringer Ingelheim and personal fees from AstraZeneca, GlaxoSmithKline, and Novartis outside the submitted work. Dr. Thomashow is co-founder and former Chair of the Board of Directors for the COPD Foundation and currently serves as its Chief Medical Officer. He has served as a consultant for Boehringer Ingelheim and served on advisory boards for GlaxoSmithKline and AstraZeneca. Dr. Wise reports grants and personal fees from AstraZeneca/Medimmune, GlaxoSmithKline, Novartis and Boehringer-Ingelheim, personal fees from Contrafect, PulmonX, Roche / Genentech, Spiration, Pneuma, Verona, Bonti, Denali, Aradigm, Mylan, Theravance, Propeller Health, Kiniksa, Sunovion, Merck, and Circassia; and grants from Pearl Therapeutics, outside the submitted work. Ms. Boyce has nothing to declare. 


\section{References}

1. COPD Foundation. COPD Foundation Medical and Scientific Advisory Council. Updated January 2020. Accessed May 2, 2020. https://www.copdfoundation.org/About-Us/Who-We-Are/ Medical-and-Scientific-Advisory-Committee.aspx

2. COPD Foundation. Coronavirus information for the COPD community. Updated April 29, 2020. Accessed May 2, 2020. https://www.copdfoundation.org/Learn-More/I-am-a-Personwith-COPD/Coronavirus-Information.aspx

3. Global Initiative for Chronic Obstructive Lung Disease (GOLD). GOLD COVID-10 guidance. Published 2020. Accessed May 2, 2020. https://goldcopd.org/gold-covid-19-guidance/

4. Centers for Disease Control and Prevention. Coronavirus disease 2019 (COVID-19). Groups at higher risk for severe illness. Published April 17, 2020. Accessed May 2, 2020. https:// www.cdc.gov/coronavirus/2019-ncov/need-extra-precautions/ groups-at-higher-risk.html

5. Russell CD, Millar JE, Baillie JK. Clinical evidence does not support corticosteroid treatment for 2019-nCoV lung injury. Lancet. 2020 Feb 15;395:473-495.

doi: https://doi.org/10.1016/ S0140-6736(20)30317-2

6. Arabi Ym, Mandourah Y, Al-Hameed, F, et al. Corticosteroid therapy for critically ill patients with Middle East respiratory syndrome. Am J Respir Crit Care Med. 2018;197(6):757-767. doi: https://doi.org/10.1164/rccm.201706-1172OC

7. Bikdeli B, Madhavan MV, Jimenez D, et al. COVID-19 and thrombotic or thromboembolic disease: implications for prevention, antithrombotic therapy, and follow-up. J Am Coll Cardiol. 2020 Apr 15; In press.

doi: https://doi.org/10.1016/j.jacc.2020.04.031

8. Phend C. COVID-19: anticoagulation recommended even after discharge. Published April 30, 2020. Accessed May 1, 2020.

https://www.medpagetoday.com/infectiousdisease/covid19/86230

9. Grillet F, Behr J, Calame P, Aubry S, Delabrousse E. Acute pulmonary embolism associated with COVID-19 pneumonia detected by pulmonary CT angiography. Radiology. 2020 Apr 10; In press. doi: https://doi.org/10.1148/radiol.2020201544

10. Fischer and Paykel Healthcare. Heated humidification for healthcare professionals managing COVID-19 patients. Published May 2020. Accessed May 2, 2020 https://resources. fphcare.com/content/heated-humidification-healthcarepractitioners-covid19-pm-620199.pdf

11. COPD Foundation. Exploring the COPD patient and caregivers experience with COVID-19 through surveys: highlights from survey \#1. Published April 22, 2020. Accessed May 2, 2020. https://www.copdfoundation.org/COPD360social/Community/ COPD-Digest/Article/1566/Exploring-the-COPD-Patient-andCaregivers-Experience-with-COVID-19-through-Surv.aspx
12. Guan W-J, Liang W-H, Zhao Y, et al on behalf of the China Medical Treatment Expert Group for COVID-19. Comorbidity and its impact on 1590 patients with COVID-19 in China: a nationwide analysis. Eur Respir J. 2020; In press. doi: https://doi.org/10.1183/13993003.00547-2020

13. Goyal P, Choi JJ, Pinheiro LC, et al. Clinical characteristics of COVID-19 in New York City. New Eng J Med. 2020 Apr 17. In press. doi: https://doi.org/10.1056/NEJMc2010419 